\title{
Pneumatic fractures in confined granular media
}

\author{
Fredrik K. Eriksen, ${ }^{*}$ Renaud Toussaint, and Antoine L. Turquet \\ Institut de Physique du Globe de Strasbourg, Université de Strasbourg/EOST, Centre National de la Recherche Scientifique, \\ 67084 Strasbourg, France
}

Knut J. Måløy and Eirik G. Flekkøy

PoreLab, Department of Physics, University of Oslo, P.O. Box 1074 Blindern, 0316 Oslo, Norway

(Received 19 October 2016; revised manuscript received 22 May 2017; published 12 June 2017)

\begin{abstract}
We perform experiments where air is injected at a constant overpressure $P_{\text {in }}$, ranging from 5 to $250 \mathrm{kPa}$, into a dry granular medium confined within a horizontal linear Hele-Shaw cell. The setup allows us to explore compacted configurations by preventing decompaction at the outer boundary, i.e., the cell outlet has a semipermeable filter such that beads are stopped while air can pass. We study the emerging patterns and dynamic growth of channels in the granular media due to fluid flow, by analyzing images captured with a high speed camera (1000 images/s). We identify four qualitatively different flow regimes, depending on the imposed overpressure, ranging from no channel formation for $P_{\text {in }}$ below $10 \mathrm{kPa}$, to large thick channels formed by erosion and fingers merging for high $P_{\text {in }}$ around $200 \mathrm{kPa}$. The flow regimes where channels form are characterized by typical finger thickness, final depth into the medium, and growth dynamics. The shape of the finger tips during growth is studied by looking at the finger width $w$ as function of distance $d$ from the tip. The tip profile is found to follow $w(d) \propto d^{\beta}$, where $\beta=0.68$ is a typical value for all experiments, also over time. This indicates a singularity in the curvature $d^{2} d / d w^{2} \sim \kappa \sim d^{1-2 \beta}$, but not of the slope $d w / d d \sim d^{\beta-1}$, i.e., more rounded tips rather than pointy cusps, as they would be for the case $\beta>1$. For increasing $P_{\text {in }}$, the channels generally grow faster and deeper into the medium. We show that the channel length along the flow direction has a linear growth with time initially, followed by a power-law decay of growth velocity with time as the channel approaches its final length. A closer look reveals that the initial growth velocity $v_{0}$ is found to scale with injection pressure as $v_{0} \propto P_{\text {in }}^{\frac{3}{2}}$, while at a critical time $t_{c}$ there is a cross-over to the behavior $v(t) \propto t^{-\alpha}$, where $\alpha$ is close to 2.5 for all experiments. Finally, we explore the fractal dimension of the fully developed patterns. For example, for patterns resulting from intermediate $P_{\text {in }}$ around $100-150 \mathrm{kPa}$, we find that the box-counting dimensions lie within the range $D_{B} \in[1.53,1.62]$, similar to viscous fingering fractals in porous media.
\end{abstract}

DOI: 10.1103/PhysRevE.95.062901

\section{INTRODUCTION}

Several processes in engineering, industry, and earth sciences involve pneumatic (gas) or hydraulic (liquid) fracturing of the soil, which occurs when fluids in the ground are driven to high enough pressures to deform, fracture, and generate porosity in the surrounding soil or rock. For example, in environmental engineering, pneumatic or hydraulic fracturing is done to enhance the removal of hazardous contaminants in the vadose zone (soil remediation) [1,2], for soil stabilization injection to ensure a solid foundation for structures [3], or in packer tests for project planning, risk assessment, and safe construction of dams and tunnels [4]. In industry, hydraulic fracturing is done to enhance oil and gas recovery [5-7], $\mathrm{CO}_{2}$ sequestration [8], water well and geothermal energy production [9-11]. Related natural processes, such as subsurface sediment mobilization, are studied in earth sciences, where sand injectites, mud diapirs, and mud volcanoes are formed due to pore-fluid overpressure [12-17]. For example, the Lusi mud volcano in Indonesia is the biggest and most damaging mud volcano in the world [18], having displaced 40000 people from their homes, and has been active since May 2006. There

\footnotetext{
*Also at PoreLab, Department of Physics, University of Oslo, 0316 Oslo, Norway; f.k.eriksen@fys.uio.no
}

is an ongoing debate about how it was triggered, i.e., whether it formed naturally by an earthquake or geothermal process [19-22], or if it is a manmade consequence of a nearby drilling operation by a company probing for natural gas [23].

Fluid injections into granular media has been extensively studied in laboratory experiments and simulations, where a common method to simplify the problem is to confine the experiment within a quasi-two-dimensional geometry, i.e., a Hele-Shaw cell. In Refs. [24,25], the decompaction, fluidization regimes, and coupling between air and granular flow was studied in dry granular media in open circular and rectangular cells during air injection at different overpressures. Similar behavior was seen for liquid saturated granular material injected with the same liquid [26], so it is reasonable to assume that studies of pneumatic fracturing also have qualitative relevance to hydraulic fracturing. The two cases are similar in the sense that no interface tension and viscosity difference exist between the invading and interstitial fluids. In systems where gravity is felt significantly, as in inclined or vertical systems, the density of the fluid is a notable difference, and buoyancy forces on the grains are significant for liquids but usually negligible for gases. In horizontal layers and thin compacted systems, where gravity is perpendicular to the system and plays no major role on the horizontal motion of such quasi-two-dimensional (quasi-2D) systems, the main difference in an experiment where a liquid is used 
instead of a gas is that the compressibility is lower such that the overpressure imposed at the boundary diffuses much faster [27]. However, near the invading channels, the pressure gradients are found to be qualitatively similar in both cases [28], leading to similar growth mechanisms for the channels [26]. Further, the compressibility felt in water on the scale of an $\sim 100-\mathrm{m}$ reservoir is similar to the compressibility felt in air on the scale of $\mathrm{a} \sim 1-$ m experiment, and therefore the effect of pressure diffusion is included when air is used in $\sim 1-\mathrm{m}$ model reservoirs. Another difference is that for a given pore pressure gradient and grain configuration (permeability), the fluid velocity between the grains is inversely proportional to the viscosity force. The stress thresholds allowing to overcome friction with the confining plates depends mainly on the solid fraction and does not change in order of magnitude between cases where the interstitial fluid is a gas or a liquid [26]. Consequently, for similar channel geometries and grain packings, the pressure difference between inlet and outlet that lead to grain motion are similar in the two systems. However, the grain mobility is observed to be essentially inversely proportional to the pore fluid viscosity; i.e., the observed dynamics are similar in terms of a reduced time corresponding to time multiplied by the pore fluid viscosity [26]. The patterns observed are also close to each other [26]. The patterns formed during fluid injection into a granular medium, and evolution of the fluid-solid interface have been found to resemble diffusion limited aggregation patterns (DLA) and viscous fingering [29], a fingering instability that occurs when a less viscous liquid is injected into a porous medium containing a more viscous liquid with which it cannot mix [30]. As mentioned in Ref. [25], the main difference between the viscous- and granular-fingering instabilities is the absence of interfacial tension in the granular case. For example, the stabilizing forces in viscous fingering are surface forces, while in granular fingering it is the build-up of friction between particles and against the confinement. However, both instabilities are driven by the pressure gradient across the defending medium, which is largest on the longest finger tips, making more advanced fingers grow on expense of the less advanced ones. A notable difference between air injection into a dry granular medium and a saturated one is that the overpressure initially diffuses into the packing in the dry case, while it is already a steady-state Laplace field over the defending liquid in the saturated case. This difference arises from the compressibility of the defending phase.

Further, during air injection into liquid saturated granular media and suspensions, the characteristics of emerging patterns and behavior of the media depend on injection rate and the competition between mobilized friction and surface forces [31-44]. For example, one observes flow regimes such as two-phase flow in rigid porous media [40-44], capillary fracturing, stick-slip bubbles, and labyrinth patterns [31-39]. In the opposite case, during liquid injection into dry granular media [45], for a given imposed flux, the flow behavior goes from stable invasion toward saturated granular fingers for increasing flow rate and viscosity of the invading fluid. These fingering patterns are thought to form due to the permeability contrast between the channels empty of grains and the granular medium and are in this sense similar to viscous fingering, with a lower viscous pressure drop in empty channels than in the porous medium, as in classical viscous-fingering systems, where the viscosity contrast leads to a similar effect, i.e., where the invading fluid has a lower viscosity than the invaded one $[30,42,46]$. An important difference with classical viscous fingering is the presence of solid stresses and solid friction in the granular material. This is a stabilizing phenomenon absent from systems with two Newtonian fluids: A threshold arising from friction with the system boundaries has to be overcome in viscous fluid drag to allow for grain motion. The same trend is shown in numerical studies for gas injection into granular media containing the same gas [47].

Granular fingering instabilities have also been studied in closed vertical cells, where gravity drives the flow as heavier beads fall down from a granular layer at the top of a lighter fluid layer [48-53]. When the beads detach at the front, they form fingers of falling granular material surrounding fingerlike bubbles of rising fluid. These fingers are found to coarsen over time until they reach a typical wavelength depending on the interstitial fluid and bead size.

Typically, in all processes involving fluid injection into granular media, there are flow regimes where the medium has either solid-like behavior or fluid-like behavior. This is one of the special properties of granular materials, which also show gas-like behavior in some cases [54].

In this paper, we present an experimental study on flow regimes and pattern formation during air injection into confined granular media, while the surrounding deformation and pore-pressure evolution is studied in a related article [28]. More specifically, we inject air at constant overpressure into a dry granular medium inside a Hele-Shaw cell, where air escapes at the outlet while beads cannot. The motivation of this setup is to study the granular Saffman-Taylor instability in compacting granular media and the coupling between compaction and flow. A similar, but smaller system has been studied in numerical simulations by Niebling et al. [47,55]. During air injection at different overpressures they found that fractures grow faster, longer, as well as coarsen with increasing injection pressure. There, the fracture propagation velocity is roughly constant initially and scales with the square root of the injection pressure. Further, by varying the interstitial fluid viscosity, two flow regimes were identified; one with finely dispersing bubbles and large-scale collective motion of particles, the other one with build-up of a compaction front and fracturing. These flow regimes depend, respectively, on whether the particles are primarily accelerated by the imposed pressure gradient in the fluid or interactions through particle contacts. This in turn depends on the diffusivity of the interstitial fluid pressure in the granular medium. We analyze our findings in light of these results to investigate what is similar and what is different in our roughly 10-timeslarger cell with the same cell gap. As opposed to similar experiments, with open outer boundary conditions, after the flow compacts the medium there is no decompaction. We thus expect the material behavior (at high enough overpressure to displace beads) to have a transition from fluid-like to solid-like during experiments, and that eventual invasion patterns will initially resemble viscous fingering in the fluid-like regime, crossing over to stick-slip fracture propagation as the medium becomes more solid-like, until it reaches a final structure as the compacted medium has reached a completely solid-like behavior. What is less obvious is how the flow patterns in 
this system change with the injection pressure. By varying the imposed overpressure, we identify and describe the different flow regimes. Due to the confined nature of the experiment, it is thought to be a laboratory analog to pneumatic and hydraulic fracturing of tight rock reservoirs where the free boundary at the surface is very distant from the injection zone. Therefore, new insight into this problem may have industrial applications in addition to increase the understanding of flow and transformations in porous media.

It is also worth to mention a closely related project [56], where acoustic emissions recorded during the experiments are analyzed. There, it is shown that different stages of the invasion process can be identified acoustically in terms of characteristic frequencies and distinct microseismic events.

\section{METHODS}

\section{A. Experimental setup}

The experimental setup is a linear Hele-Shaw cell, partially filled with Ugelstad spheres [57], i.e., dry, nonexpanded polystyrene beads with a diameter of $80 \mu \mathrm{m} \pm 1 \%$. The cell is made out of two rectangular glass plates $(80 \times 40 \times 1 \mathrm{~cm}$ in length, width, and thickness, respectively) clamped together on top of each other with an aluminum spacer controlled separation of $1 \mathrm{~mm}$. A cell volume $(76 \times 32 \times 0.1 \mathrm{~cm})$ is formed between the plates by an impermeable sealing tape as shown in Fig. 1, with one of the short sides left open (outlet). Next, beads are filled into the cell by pouring them through the open side until the packing occupy about $90 \%$ of the cell volume, followed by closing the open side with a semipermeable filter (a 50- $\mu \mathrm{m}$ steel mesh) to keep beads inside the cell while allowing air to escape. The cell is then flipped vertically to place the granular medium against the semipermeable outlet by using gravity, resulting in a volume-packing fraction of approximately $\rho_{s}=0.44 \pm 0.04$, assumed to be more or less uniform across the medium (in a quasi-2D system of $\sim 10$ beads thickness, the confining plates cause a frustrated system with lower packing fraction [58], as observed previously in similar systems where $\rho_{s}=0.44$ [24] and $\rho_{s}=0.42$ [47]). This leaves a volume empty of beads on the sealed side of the cell, opposite to the semipermeable outlet, with a linear air-solid interface. An inlet hole on the sealed side of the cell is connected to a pressurized air tank, which lets us inject air at a constant and maintained overpressure, $P_{\text {in }}=P_{\text {abs,in }}-P_{0}$ (absolute pressure - atmospheric pressure), ranging from 5 to $250 \mathrm{kPa}$. This will force air to move through the granular medium, toward the semipermeable outlet, where $P_{\text {abs,out }}=P_{0}=100 \mathrm{kPa}$, or in terms of overpressure above the atmospheric one, $P_{\text {out }}=0$.

During experiments, the prepared cell is positioned horizontally. A selected overpressure is set at the pressure tank outlet and verified by a Honeywell pressure sensor with an accuracy of $\pm 4 \mathrm{kPa}$. The tubing between the pressure tank and the cell inlet is equipped with an electronic valve such that the air injection is started with a digital trigger signal. Positioned above, with a top-down view of the cell, a Photron SA5 high-speed camera is started with the same trigger signal, recording the air invasion at a frame rate of 1000 images $/ \mathrm{s}$ and a resolution of $1024 \times 1024$ pixels $(1$ pixel $\approx 0.7 \mathrm{~mm}$ in (a)

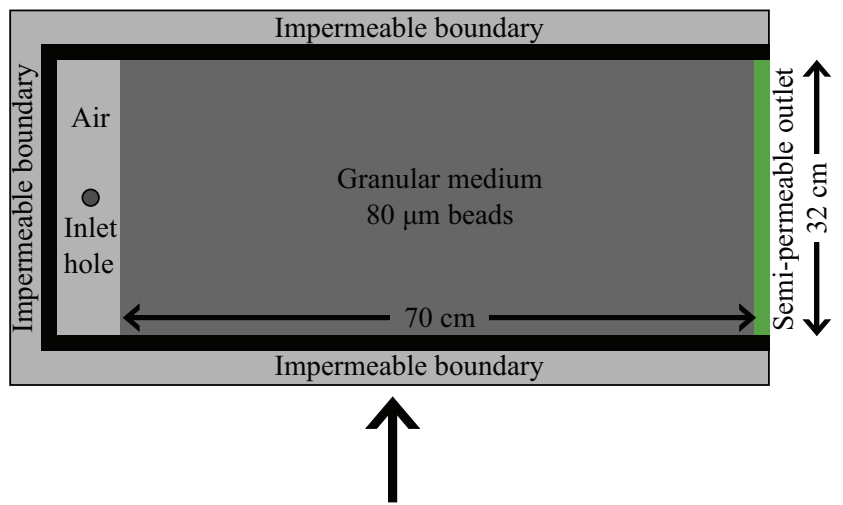

(b)

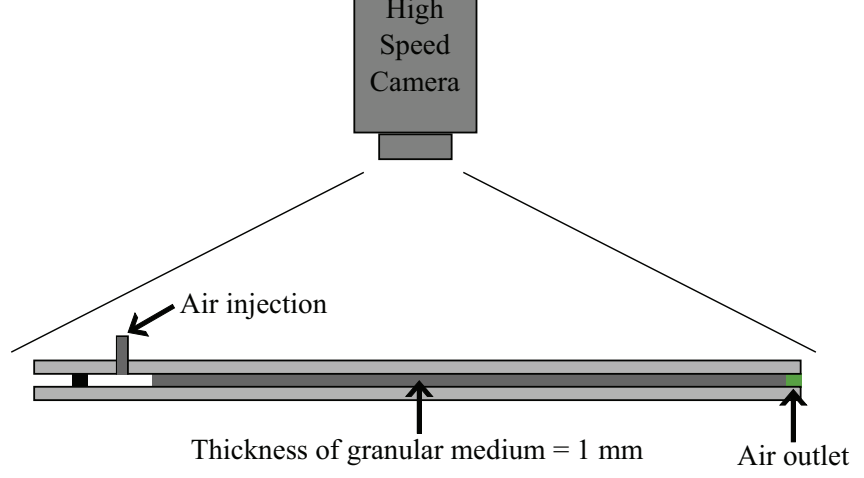

FIG. 1. Sketch of the experimental setup. (a) Top-down view of the prepared cell: The granular medium is confined inside the cell by three impermeable boundaries and an air-permeable boundary on the outlet side. The granular medium is placed against the outlet boundary such that it has a linear interface against a region empty of beads on the sealed inlet side, where pressurized air can be injected. (b) Side view of the setup showing the high speed camera placed above. The glass plates are clamped together with aluminum framing while the cell gap is controlled with spacers, which are not shown here.

the cell). Light from a $400 \mathrm{~W}$ Dedolight studio lamp provides uniform and flicker-free illumination onto the white beads of the medium. A small fraction of the beads $(<10 \%)$ are dyed black with ink to create tracer particles that are used for tracking frame-to-frame deformation in the granular medium, which is studied in another article [28] (note that in Figs. 2, 3 , and 6 , there are some darker areas creating patterns in the initial packing. This is due to a higher concentration of dyed beads, which is related to the initial preparation process and the packing method. It does not seem to have any influence on the experiments). The experiments are run for $10 \mathrm{~s}$, but typically the fracturing and/or compaction of the granular medium takes less than $5 \mathrm{~s}$.

\section{B. Image processing}

In analysis of the images from the high-speed camera, we investigate flow regimes, characteristics, and evolution of the fingering patterns formed. We perform image processing with Matlab to obtain the information contained in the images. Quantitative analysis of the invasion patterns is done by converting the gray-scale raw data into binary images, i.e., 

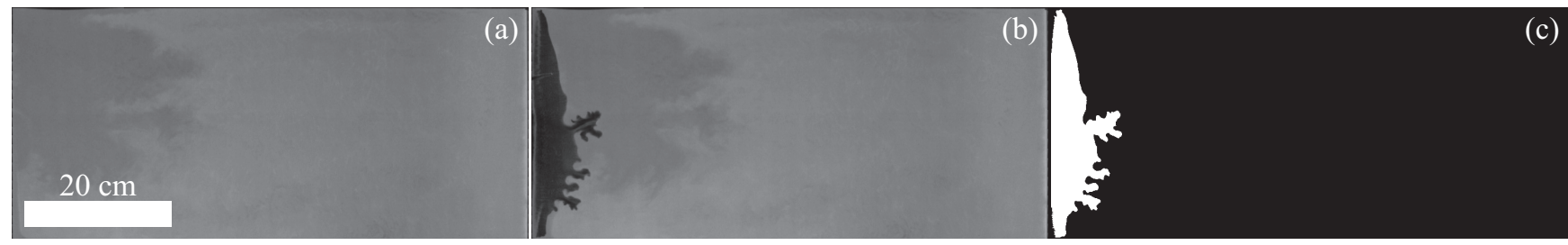

FIG. 2. Example of image processing. (a) The initial granular medium, (b) a snapshot during the experiment, (c) the resulting binary image where the pattern has been segmented. The segmentation is done by setting all pixels having $30 \%$ less gray-scale intensity in a snapshot compared to the initial image to the value 1 while the other pixels are set to the value 0 .

images with either black or white pixels, where the white pixels (value $=1$ ) represent the pattern and the black pixels (value $=0$ ) represent the background, or the bead-filled region. We obtain such binary image sequences from the raw data by thresholding each frame with the initial image, such that the pixels having a value less than $30 \%$ of the corresponding initial value becomes white and the rest remain black, as shown in Fig. 2(c). In addition, once a pixel is invaded (and made white) it will remain white for the rest of the experiment to correct for mobilized beads flowing inside channels, which occurs due to erosion and fingers merging in some experiments. From the binary images we extract information such as invasion depth over time, average finger thickness as function of depth, and fractal dimensions.

\section{RESULTS}

Depending on injection pressure (and friction related to packing fraction) we observe the granular medium to exhibit either a solid-like behavior or a transition from fluid-like to solid-like behavior during air injection. In the solid-like regime, there is no apparent deformation (bead displacements), and the air is reaching the cell outlet by seeping through the network of pores between beads. On the other hand, in the fluid-like regime, we observe significant deformation, where beads are displaced by an amount corresponding to several bead sizes, by momentum exchange between air molecules and beads. At sufficient overpressure the granular medium has a behavior much like a viscous liquid being invaded by air, as the flow opens up channels empty of beads, a SaffmanTaylor-like instability [30] resulting in dendritic invasion patterns. A stabilizing mechanism of this flow instability is the build-up of friction and stress chains between beads during compaction, so due to the boundary conditions in our experiments we always end up with a solid-like medium toward the end of an experiment. Since the channels empty of beads display patterns with finger-like features, and that the air-solid interface expands similarly to a fingering instability, we refer to the branches of the channels as fingers when we characterize the patterns. At the same time, the fingers have a long and slender morphology, which is fracture-like, and during growth frictional "bonds" between the compacting grains and the confining plates are broken in order to open the channels. Therefore, we consider the channel growth as fracturing, although there are no cohesive bonds broken as in the traditional sense of fracturing.

\section{A. Flow regimes}

To explore the various flow regimes, we have systematically varied the air injection pressure within the range of 5-250 kPa, and we have identified four qualitatively different flow regimes occurring in this range. Within certain flow regimes, the pattern characteristics gradually change with increasing injection pressure, so we have grouped the experimental results into six categories defined by final pattern characteristics and how the channels expand. The categories are chosen in order to obtain average results from similar experiments and are defined in the list below. Typical final structures are shown for each category in Fig. 3, and the associated range of injection pressure is listed in Table I. Videos of channels formed in categories $3 \mathrm{a}, 3 \mathrm{~b}$ and $4 \mathrm{a}, 4 \mathrm{~b}$ are found in the Supplemental Material [59].
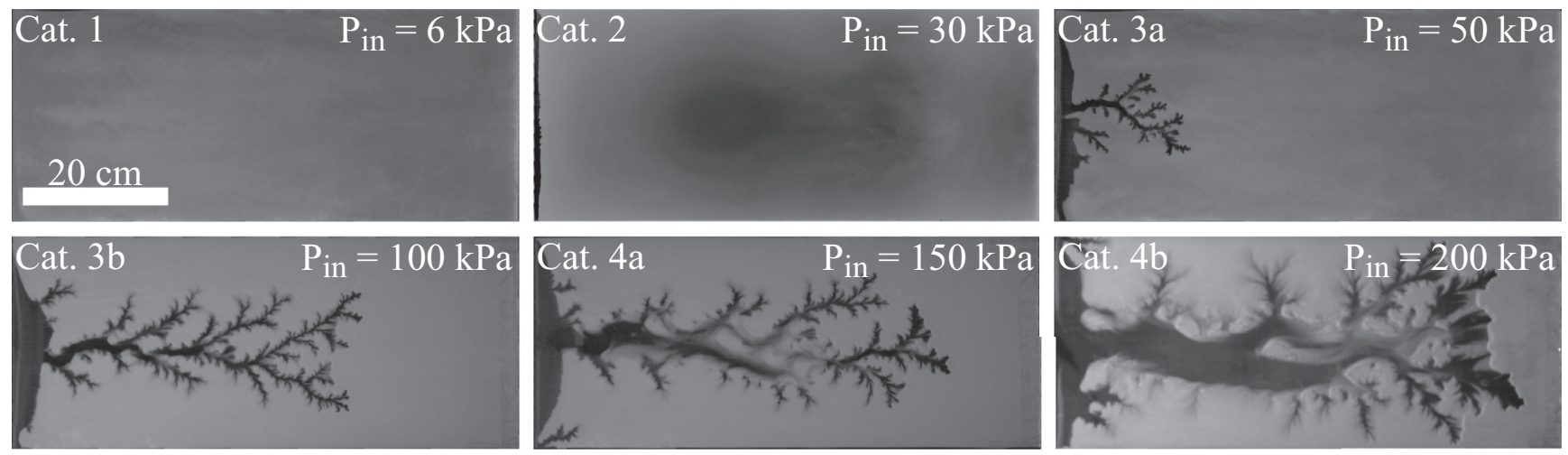

FIG. 3. Typical final structures per category, where the granular medium is gray and areas empty of beads are black, showing that for increasing $P_{\text {in }}$ the fingers become longer and thicker. A full-size figure of the patterns shown here is found in the Supplemental Material [59]. 
TABLE I. The flow categories with related pressure values; mean overpressure within two standard deviations $\overline{P_{\text {in }}} \pm 2 \sigma$, as well as the number of experiments $N$ analyzed per category.

\begin{tabular}{lcr}
\hline \hline Category & $\overline{P_{\text {in }}} \pm 2 \sigma[\mathrm{kPa}]$ & $N$ \\
\hline 1 & $9 \pm 5$ & 3 \\
2 & $26 \pm 18$ & 5 \\
$3 \mathrm{a}$ & $64 \pm 50$ & 5 \\
$3 \mathrm{~b}$ & $110 \pm 44$ & 6 \\
$4 \mathrm{a}$ & $134 \pm 43$ & 10 \\
$4 \mathrm{~b}$ & $213 \pm 50$ & 4 \\
\hline \hline
\end{tabular}

(i) Category $1-$ No deformation: The porous medium appears rigid (solid-like), with no significant bead displacement or invasion pattern formed. This is flow regime \#1, where the pore pressure gradient does not overcome the threshold for bead displacement, which arise from granular stress, friction between the beads, and bead-plate friction.

(ii) Category 2-Initial compaction without channels: The medium is initially compacted during a collective displacement of beads toward the outlet side, without the formation of invasion channels. This is flow regime \#2, where the pore pressure gradient is initially high enough to overcome the granular stress threshold arising from the friction with the plates. Then, as the pore pressure diffuses into the medium, the pore pressure gradient drops below the bead displacement threshold and the deformation stops before channels are formed. During the short time beads are displaced, they are primarily moved by the pore pressure gradient, i.e., stress from the fluid injection transmitted through solid contacts (compacting zone) does not significantly extend beyond the influence of the overpressure (skin depth), leading to collective bead displacement rather than the formation of fractures [47].

(iii) Categories $3 \mathrm{a}$ and $3 \mathrm{~b}$-Thin branched channels are formed: After the initial compaction, a dendritic invasion pattern is formed with an average finger thickness less than 2 $\mathrm{cm}$. This is flow regime \#3, where the pressure gradient is high enough to deform the medium, also after the compacting zone reaches beyond the influence of the pore pressure gradient. This leads to random perturbations in the air-solid interface and fracture channels begin to form [47]. At later times, the channel growth stops as the medium is compacted, i.e., the solid stress and friction increase such that the bead displacement threshold eventually becomes too large for further deformation. In this flow regime, the channels become longer with increasing injection pressure, so we define arbitrarily (in order to perform statistical analyses on groups of experiments) category $3 \mathrm{a}$ as when the final length of the channel is less than $50 \%$ of $L=70$ $\mathrm{cm}$, the initial length of the granular medium, and we define category $3 \mathrm{~b}$ as when the final length of the channel is more than $50 \%$ of $L$.

(iv) Categories $4 \mathrm{a}$ and $4 \mathrm{~b}-$ Branched channels are formed and eroded: After the initial compaction, a long dendritic invasion pattern is formed (as in category $3 \mathrm{~b}$ ) with a main channel thicker than the branch fingers, due to erosion inside the channel. This is flow regime \#4, where the high pressure gradients at the most advanced channel tips cause a large enough fluid flux inside the channels to remobilize beads along with the flow. The width of the main channel increases by erosion (i.e., motion of beads nonperpendicular to the channel interface, but with a major tangential component along it). For higher pressures, side channels and branches may also merge into the main channel. In this case, the high flow velocity in the main channel might reduce the fluid pressure slightly such that adjacent channels grow into it. In this flow regime, the degree of erosion increases gradually with increasing injection pressure, so we define two categories. Category $4 \mathrm{a}$ is defined as when there is only erosion, where the average finger thickness of the final structure is typically between 2 and $4 \mathrm{~cm}$. The erosion is not persistent and rarely occurs more than $15 \mathrm{~cm}$ behind the most advanced tip. Category $4 \mathrm{~b}$ is defined as when there is both erosion and channels merging, where the average finger thickness of the final structure is typically more than $4 \mathrm{~cm}$. Erosion and channel merging is observed to occur even at distances of $30-40 \mathrm{~cm}$ behind the most advanced tip. Again, the erosion is not persistent and stops around the point when the channel structure reaches $90 \%$ of its final length.

As one can expect due to the heterogeneous nature of the grain packings, and the variability of the grain assembly between experiments, a significant dispersion of the resulting patterns is observed for experiments at similar imposed pressures, and consequently, the pressure ranges for the categories overlap (see Table I). The distribution of new growth $d A$ behind the longest fingers at different intervals is shown in figure 4 for typical experiments in the categories $3 \mathrm{a}, 3 \mathrm{~b}$ and $4 \mathrm{a}, 4 \mathrm{~b}$. The new growth $d A$ refers to the change in channel area between two successive snapshots. The new growth in Fig. 4 is found by subtracting successive binary images $A$ of the patterns pixel by pixel, i.e., $d A=A(t+\Delta t)-A(t)$, where $\Delta t$ is the time between snapshots where the channels grow by $10 \%$ increments of their final lengths (this method of selecting $\Delta t$ is done in order to determine where the channels are growing at different stages, and thus to plot distributions of $d A$ with comparable space integrals at different times of the experiment, since the growth rate decreases at later times). In the plots, the distribution of new growth over the distance $d$ behind the most advanced finger is represented by a normalized value $n_{d A}(d)$, which is the mass of the new growth $d A$ at the depth $d$, divided by the width of the cell such that $n_{d A}(d)$ is a dimensionless number $\in[0,1]$, indicating the fraction of the cell width at position $d$ that contains new growth. For categories $3 \mathrm{a}, 3 \mathrm{~b}$, and $4 \mathrm{a}$ the growth typically extends in a region of $10-15 \mathrm{~cm}$ behind the most advanced tip, whereas for category $4 \mathrm{~b}$ the growth extends along the whole channel length. The category $4 \mathrm{~b}$ channels have a rapid and large volume change compared to the other ones, with a high air flow velocity resulting in erosion along the entire channel. In addition, the rapid expansion could let the channel pressure remain somewhat lower than $P_{\text {in }}$ during growth, such that when it stops the pressure rises to $P_{\text {in }}$. If it is so, pressure gradients could form at the solid-air interface far behind the most advanced finger explaining the small growth there. The displacement of the granular medium outside the channels is similar for all categories; Beads are pushed in front of the channels and compacted until the system is jammed. Beads are also pushed out from the side of the advancing channel tips, opening up for branches.

In the rest of the paper, we focus on the categories $3 \mathrm{a}, 3 \mathrm{~b}$ and 4a, 4b, which are in flow regimes \#3 and \#4, where invasion 

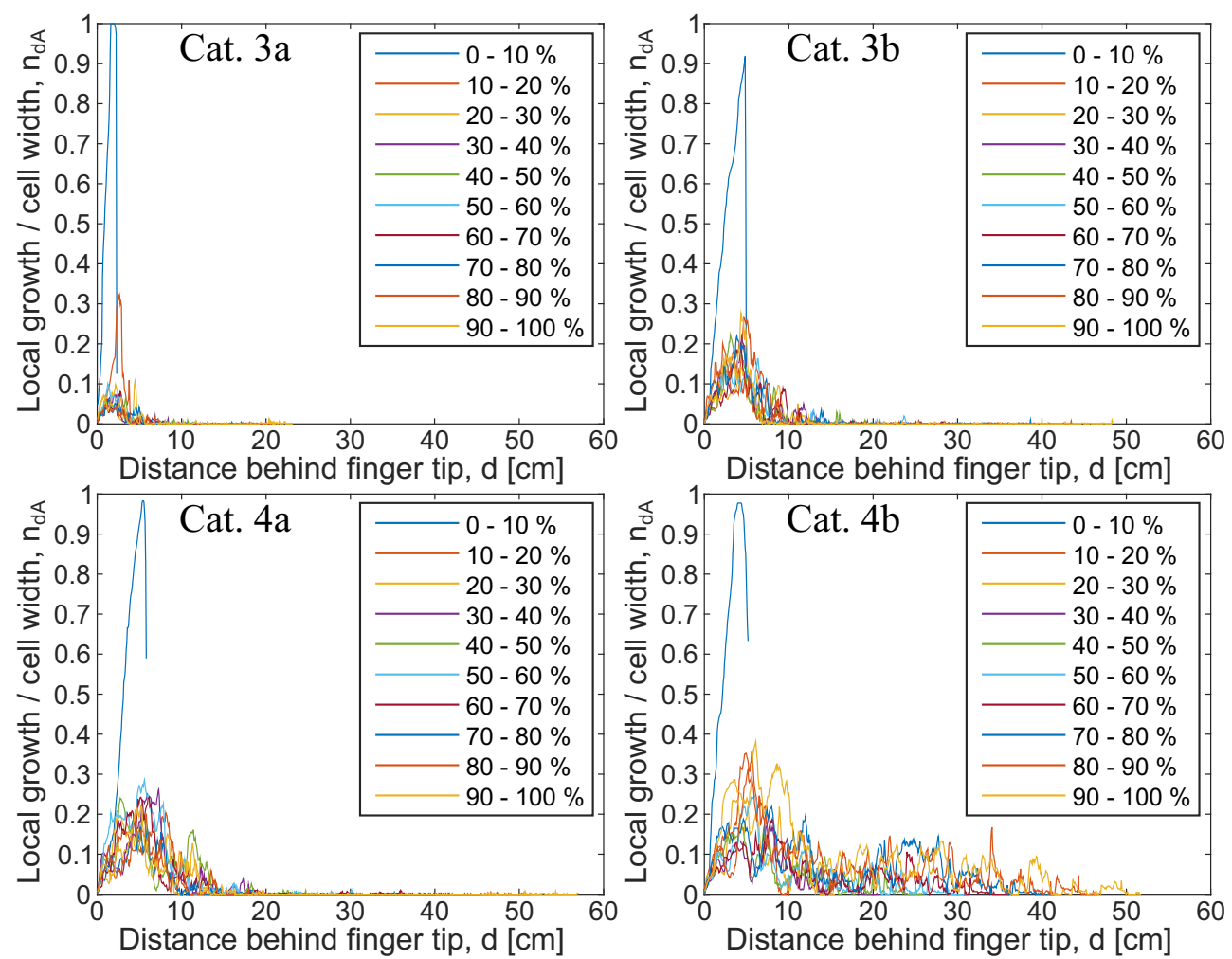

FIG. 4. Distribution of new growth over the distance behind the most advanced finger tip, at different intervals, during typical experiments in categories $3 \mathrm{a}, 3 \mathrm{~b}$ and $4 \mathrm{a}, 4 \mathrm{~b}$. The new growth is measured on intervals between successive snapshots where the channel length grows $10 \%$ of the final channel length for each snapshot. The large initial new growth (0-10\%) in all plots corresponds to the initial bulge that forms before the channeling instability begins. The plots indicate the depth of the active growth zone, which goes from very narrow for category 3 a (the growth is focused on the most advanced tips), to the whole channel length for category $4 \mathrm{~b}$.

channels are formed. For these flow regimes, we discuss the typical characteristics of the patterns formed and their growth dynamics.

\section{B. Finger characteristics}

In Fig. 5 we see the average finger thickness $w$ of the final structures as function of depth $x$ into the granular medium, measured from the initial boundary position. The average finger thickness of a pattern at a given depth into the granular medium is found by intersecting the binary image of the pattern with a line perpendicular to the average flow direction at that depth and count the total number of white pixels intersected. In other words, the total width of the pattern is measured followed by dividing it by the number of connected pixel groups, i.e., the number of fingers. This is done for each experiment and the results are averaged within each category. We see that the patterns in categories $3 \mathrm{a}$ and $3 \mathrm{~b}$ have roughly the same thickness, averaging around $1.4 \mathrm{~cm}$ and is more or less constant as function of depth. We do not observe erosion inside these channels, so a finger thickness around $1.4 \mathrm{~cm}$ seems typical when there is no erosion. The category 4a patterns show initially thicker fingers along the first half of the structures, here with an average thickness of $3.3 \mathrm{~cm}$, which then decreases down toward the typical thickness seen for categories $3 \mathrm{a}$ and $3 \mathrm{~b}$. The larger initial finger thickness is due to erosion inside the main channel, meaning that beads near the channel walls are remobilized in the flow direction after the initial growth, making the channel grow perpendicular to it. As average thicknesses here suggest, this effect typically increase the channel width by an amount of around $2 \mathrm{~cm}$. The category $4 \mathrm{~b}$ patterns typically have thick fingers throughout their entire length up to the tips, with an average of $5.3 \mathrm{~cm}$. This thickness is achieved both by erosion inside the main channel and with

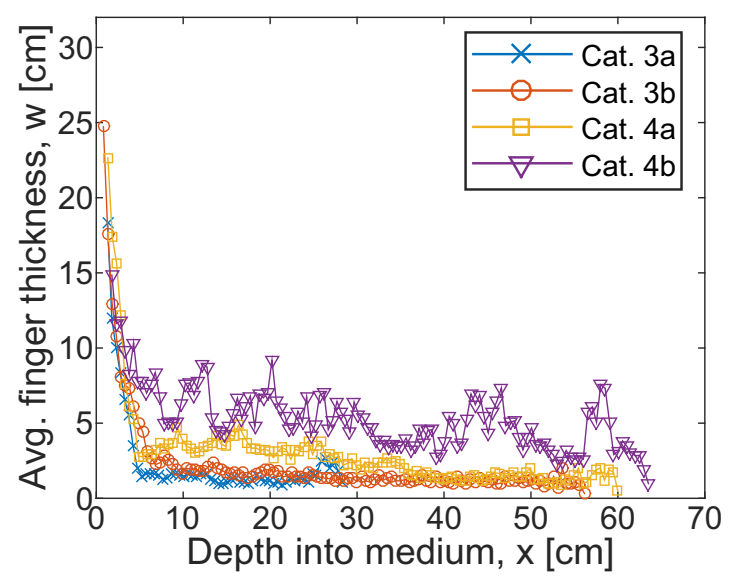

FIG. 5. Average finger thickness $w$ as function of depth $x$ into the medium for the final structures, averaged per category where channels occur. We see that for the structures in category 4 a (erosion) and category $4 b$ (erosion and finger merging) the fingers are thicker-all the way up to the finger tip for category $4 \mathrm{~b}$. 

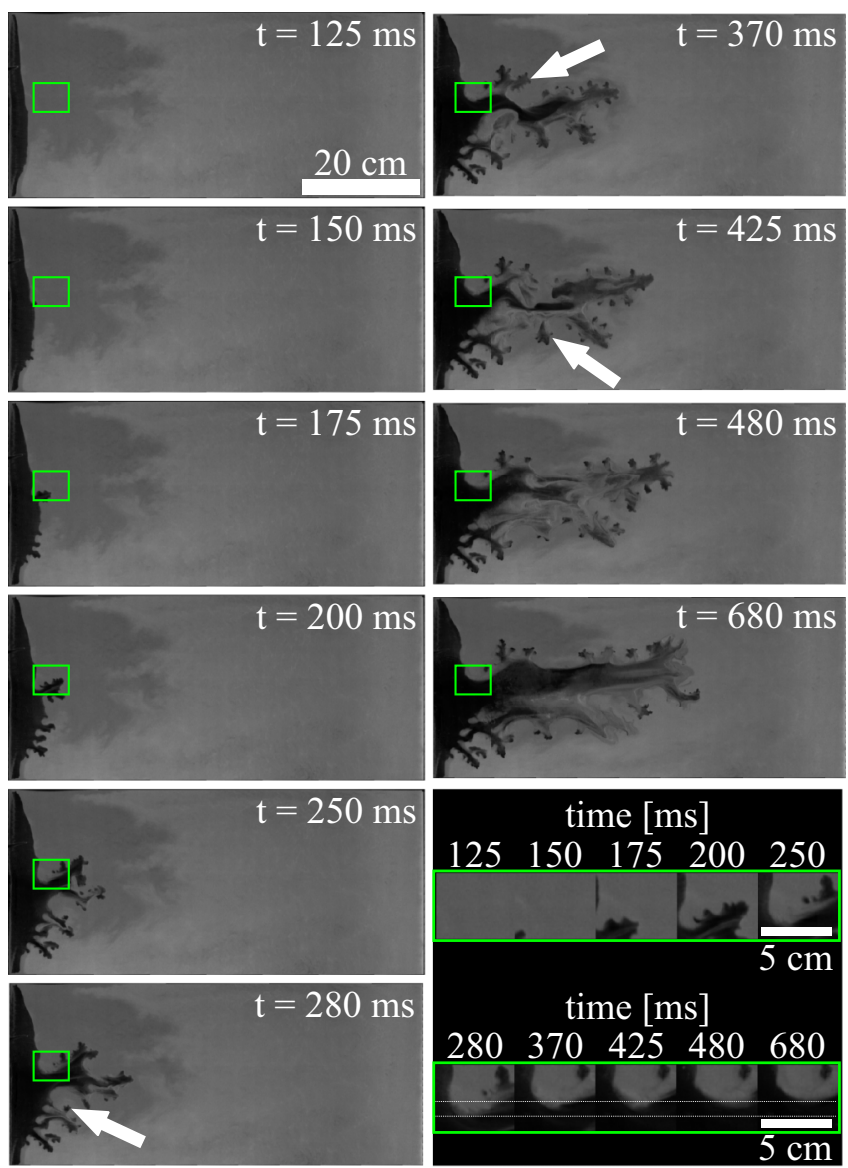

FIG. 6. Snapshots from an experiment with $P_{\text {in }}=200 \mathrm{kPa}$, showing examples of erosion and finger merging. The green rectangle indicates the area cut out and enlarged in the bottom right inset: Here we see erosion inside the channel as beads (gray area) are removed over time. The white arrows point at fingers that will merge into the main channel in the following snapshot, carving out trapped clusters of beads which are then mobilized in the flow direction. A full-size version of this figure is found in the Supplemental Material [59].

fingers merging together. After fingers merge and trap clusters of beads, these beads are mobilized in the flow direction. In general, we see an increase in finger thickness and length for increasing pressures. Some examples of both erosion and finger merging are shown in Fig. 6 for an experiment with $P_{\text {in }}=200 \mathrm{kPa}$.

The plot in Fig. 7 shows the average number of fingers $N_{f}$ as function of depth $x$ into the medium (found as the number of connected pixel groups on vertical cross-sections, as explained for Fig. 5) for the final structures in each category. For patterns in the categories $3 a$ and $3 b$, the number of fingers increase at lower depths than for patterns in the categories $4 \mathrm{a}$ and $4 \mathrm{~b}$, where there is a more pronounced initial region with fewer fingers, crossing over to more fingers during the last half of their length. This could indicate that channels with few branches form when the friction is low compared to the driving force $\propto \nabla P$, with a crossover to the patterns branching out with more fingers as the friction is becoming comparable to the driving force due to compaction. The sudden drop in $N_{f}$

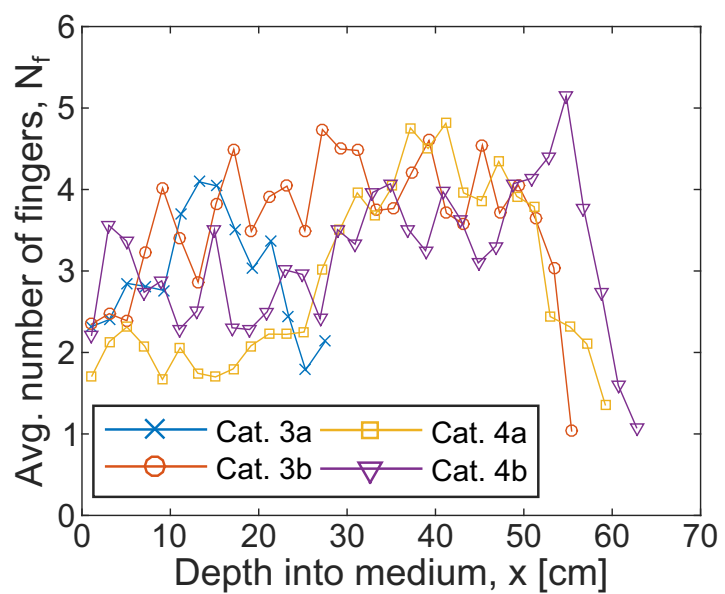

FIG. 7. The average number of fingers $N_{f}$ as function of depth $x$ into the granular medium, averaged over the final patterns in the same category. The plot indicates that for increasing injection pressure, an initial region with fewer fingers extends deeper into the medium before an increase in $N_{f}$ toward the final depth. This suggests more branching of the channels as the beads become harder to displace. The sudden drop in $N_{f}$ close to the tips is due to the finite size of the patterns.

at the deepest parts of the patterns is due to the finite size of the structures, where only a few of the longest fingers reach.

To get a description of the shape of finger tips, we look at how the finger thickness $w$ grow as function of distance $d$ from the finger tip, which is found to follow a power law within the length of the tip region [29] (where the finger thickness increase with $d$ before it saturates):

$$
w(d) \propto d^{\beta} .
$$

When $w$ is plotted as a function of $d$ in a log-log plot, the slope $\beta$ for $w(d)$ within the tip region reveal information about the shape of the tip; it has a more rounded shape for $\beta<1$, while it has a pointy cusp-shape for $\beta>1$. We have averaged $w(d)$ over time for each experiment, i.e., obtaining the average finger tip shape during flow in each experiment. Figure 8(a) shows a log-log plot of the finger thickness as function of distance from the finger tip, averaged per category. The collapsed average slopes from all experiments seem to fall along the same line with $\beta=0.68$, indicating more rounded finger tips with the same shape in all experiments. However, a purely round profile would give $\beta=0.5$, so the fact that we measure a bigger value means that there still is a singularity in curvature toward the tip-even if it is not a spike shape. The singularity can be shown as follows, where

$$
w \sim d^{\beta} \Rightarrow d \sim w^{\frac{1}{\beta}} \Rightarrow \frac{d d}{d w} \sim w^{\frac{1}{\beta}-1},
$$

gives the slope of the front. From this we find the expression for the curvature as

$$
\kappa \sim \frac{d^{2} d}{d w^{2}} \sim w^{\frac{1}{\beta}-2}\left(\text { or } d^{1-2 \beta}\right) .
$$

If $\beta>0.5, \frac{1}{\beta}-2<0$, and $\kappa$ diverges at the tip where $w($ and $d) \rightarrow 0$. At the same time, if $\beta<1$, the slope does not diverge at $d=0$ and yields a more rounded profile. 

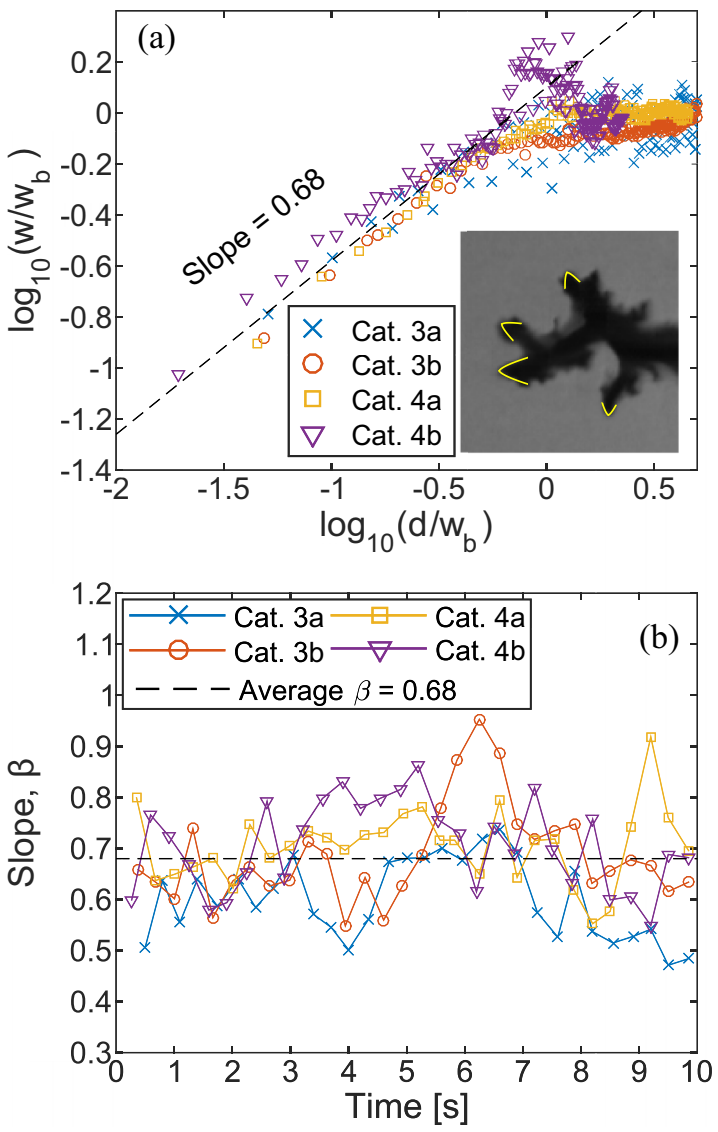

FIG. 8. (a) The finger thickness $w$ plotted in a log-log plot as function of the distance $d$ from the finger tip. The data shown is the average of about 50 measurements in time for each experiment, averaged again for each category. The data is collapsed by the finger thickness at the base of the tips $w_{b}$ found as the average finger thickness where $w$ has reached a more or less constant level, which seems to be a typical length scale for the tip region. The average slope at different times during several experiments is $\beta=0.68$, indicating a more rounded shape of the tips. In the inset: Snapshot of typical finger tips together with profiles corresponding to $\beta=0.68$ (yellow lines). (b) The slope $\beta$ averaged per category as function of time during experiments. The plot shows that the slope for the tip profile fluctuates around $\beta=0.68$ over time during the experiments, suggesting that the tips are more rounded than cusp-like at all times.

We have not observed any cusps as reported in Ref. [29] with $\beta=1.43 \pm 0.2$, consistent with theory on fluid fingering in the zero-surface tension limit. The evolution of $\beta$ over time is plotted in Fig. 8(b), which shows that for all experiments, $\beta$ fluctuates around the estimated value $\beta=0.68$ over time, indicating that the tip shapes does not seem to change significantly during the different stages of the experiments.

\section{Fractal analysis}

By looking at the invasion patterns, we notice that they have self-similar features, e.g., a smaller branch resembles the whole larger pattern. To characterize this feature, we analyze fractal dimensions of the final patterns in three different ways. The box-counting dimension $D_{B}$ is found for each final structure by covering the binary image with boxes, i.e., dividing the image into equal squares of sides $s$, and count the number $N$ of squares that contain a white pixel as function of box size $s$. For a fractal, the relationship between the number of boxes covering the pattern and their size follow a power law,

$$
N(s) \propto s^{-D_{B}}
$$

such that the box-counting dimension $D_{B}$ is found as the negative value of the slope of $N(s)$ in a log-log plot $[31,46,60,61]$. By obtaining box-counting data over a range of sizes $s$, we estimate $D_{B}$ from the slope of linear fits between an upper cutoff $s=32 \mathrm{~cm}$ (cell width) and a lower cutoff $s=1 \mathrm{~cm}$ (typical for thinner fingers). In addition, we obtain the box-counting dimension $D_{F}$ of the front (air-solid interface). This is found in the same way as the box dimension, but with binary images where only pixels on the perimeter of the patterns are white. Finally, we estimate local fractal dimensions $D_{L}$ as function of depth along the structures. To get the local fractal dimension at a given depth, we intersect the structure at that depth with a vertical line (perpendicular to the flow direction) and do a one-dimensional box counting along that line, i.e., divide the line into pieces of equal length $l$ and count the number $N$ of line segments containing white pixels as function of $l$. Again, for a fractal we have the power law,

$$
N(l) \propto l^{-D_{L}},
$$

and we find the local fractal dimensions at given depths from slopes of linear fits between $l=16 \mathrm{~cm}$ and $l=1 \mathrm{~cm}$. To compare $D_{L}$ with $D_{B}$ we use one of Mandelbrot's rules of thumb [61,62]. It states that the codimension of an intersected set equals the sum of the codimensions of the individual intersecting sets, here given by

$$
\begin{aligned}
E_{2}-D_{L} & =\left(E_{2}-E_{1}\right)+\left(E_{2}-D\right) \\
& \Downarrow \\
D & =D_{L}+1,
\end{aligned}
$$

where $D$ is the fractal dimension of the pattern, $E_{1}=1$ is the dimension of the line intersecting it, and $E_{2}=2$ is the dimension of the image plane containing the sets.

The box-counting dimensions are averaged per flow category and plotted in Fig. 9. We see that the box dimension for the less developed category 3 a patterns is $D_{B}=1.41$. For categories $3 \mathrm{~b}$ and $4 \mathrm{a}$, the box dimensions are $D_{B}=1.54$ and $D_{B}=1.53$, respectively. For category $4 \mathrm{~b}$ patterns, we observe $D_{B}=1.63$. Patterns in categories $3 \mathrm{~b}$ and $4 \mathrm{a}$ have box dimensions within the range of fractal dimensions found for viscous fingers in saturated porous media; i.e., $D=1.53-1.62$ $[31,46,63]$.

Figure 10 shows the results of box-counting the air-solid interface of the final structures, averaged per category. We see that categories $3 \mathrm{a}, 3 \mathrm{~b}$, and $4 \mathrm{a}$ have interface dimensions $D_{F}=1.40,1.52,1.48$, respectively, which are close to their corresponding box-counting dimensions, while category $4 \mathrm{~b}$ patterns have $D_{F}=1.41$, which is about 0.2 less than the corresponding box dimension. This can be interpreted in terms of finger thickness, i.e., the front becomes more similar to the structure for decreasing finger thickness. 


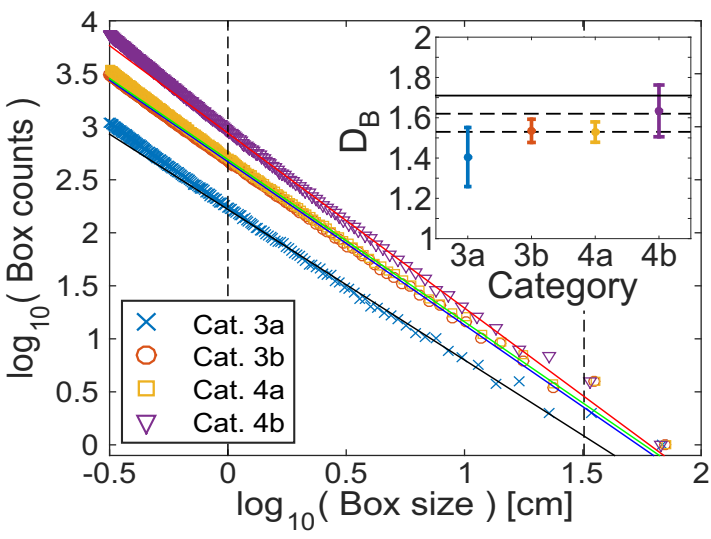

FIG. 9. Average box-counting slopes per category, the lower and upper limits are indicated with the vertical lines and mark the typical thickness of the thinnest fingers $(1 \mathrm{~cm})$ up to the cell width $(32 \mathrm{~cm})$. In the inset: Box-counting fractal dimensions $D_{B}$ averaged per category, the error bars show 2 standard deviations. Patterns in categories $3 \mathrm{~b}$ and 4 a with $D_{B}=1.53$ and $D_{B}=1.54$ are within the range of earlier measured fractal dimensions for viscous fingers in saturated porous media (between the dashed lines). The less developed patterns in category $3 \mathrm{a}$ is below this range, with $D_{B}=1.41$, and the thicker category $4 \mathrm{~b}$ patterns are close above with $D_{B}=1.63$. The solid line indicates $D_{B}=1.71$ for diffusion limited aggregation patterns.

Figure 11 shows the local fractal dimensions $D_{L}$ as function of depth into the granular medium. For all flow categories, the curves show a more or less constant behavior over most of the depths (the initial high dimension is due to a larger initial area empty of beads, and the final lower dimension is due to finite size effects of the patterns), which indicates that the patterns have consistent local fractal dimensions over a range of $x$. The categories $3 \mathrm{a}, 3 \mathrm{~b}$, and $4 \mathrm{a}$ patterns have typical local dimensions of $1.53,1.58,1.60$, respectively. This is within the range of dimensions seen for viscous fingers in saturated porous media

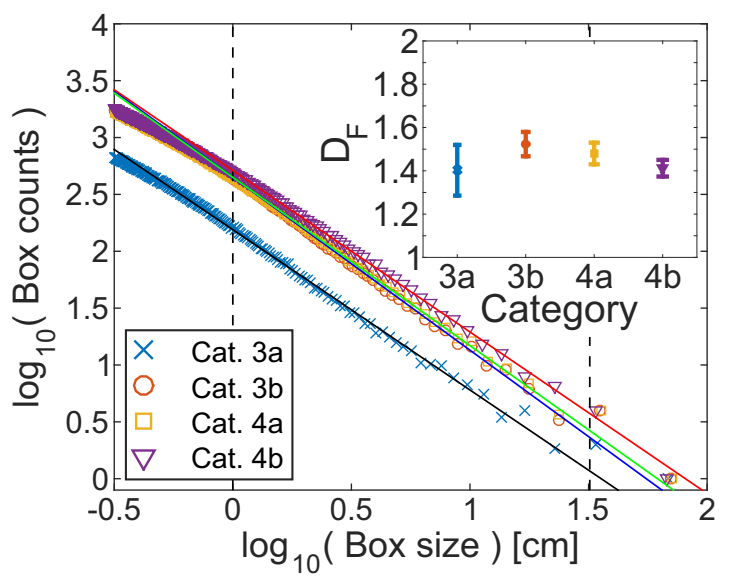

FIG. 10. Average box-counting slopes of the air-solid interface of the final structures per category, the lower and upper limits are indicated with the vertical lines and mark the typical thickness of the thinnest fingers $(1 \mathrm{~cm})$ up to the cell width $(32 \mathrm{~cm})$. In the inset: Box-counting fractal dimensions $D_{F}$ of the solid-air interface per category. The error bars show 2 standard deviations.
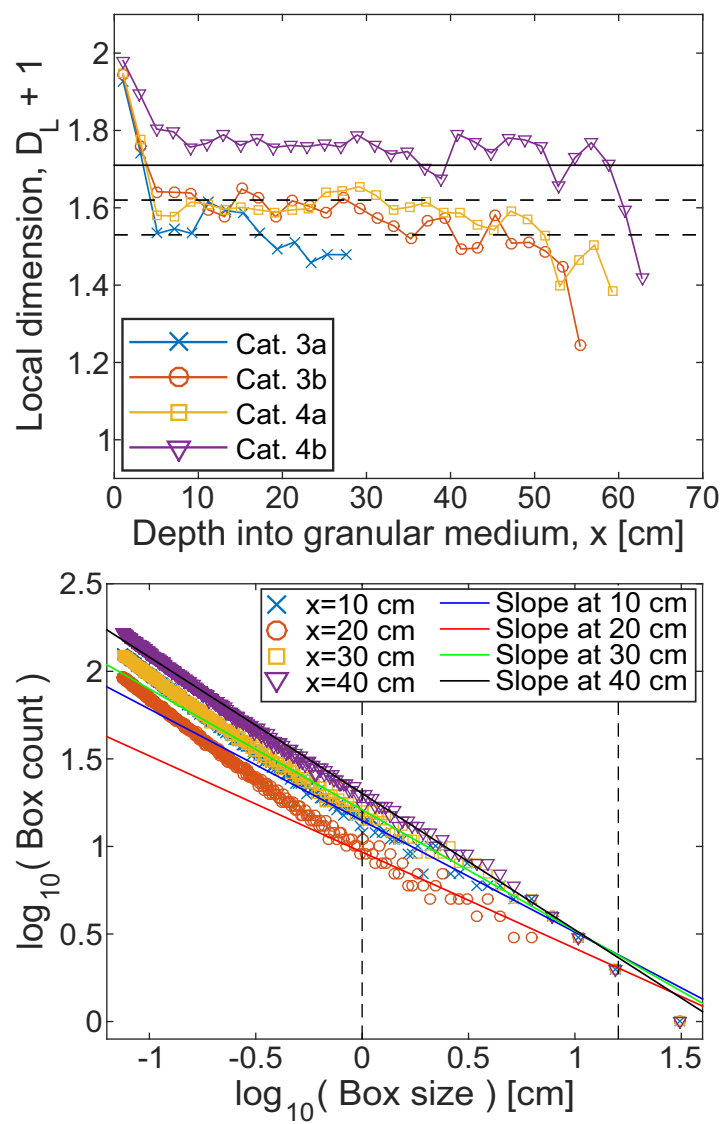

FIG. 11. Top: Local fractal dimensions $\left(D_{L}+1\right)$ as function of depth into the medium for the final structures averaged per category. We see that the patterns in all categories have more or less constant and well defined local dimensions as functions of depth. The main parts of the patterns in categories $3 \mathrm{a}, 3 \mathrm{~b}$, and $4 \mathrm{a}$ have local dimensions corresponding to established values for viscous fingers in saturated porous media (between the dashed lines). For patterns in category $4 \mathrm{~b}$, the local dimensions correspond to values closer to the fractal dimension for DLA patterns and flow patterns in a saturated HeleShaw cell (solid line). Bottom: Examples of local box-counting slopes at different depths of the same experiment.

( $D=1.53-1.62$ ), while for the category $4 \mathrm{~b}$ patterns the local dimension is higher with a typical value of 1.76 , which is closer to the dimensions seen for DLA clusters and viscous fingers in an empty saturated Hele-Shaw cell $(D=1.71)$.

\section{Growth dynamics}

In Fig. 12(a), the finger tip position averaged per category is plotted as function of time, which is found by recording the maximum depth of the patterns in the binary images at each snapshot. We see that for all experiments, the channel length grows linearly with time initially before it decelerates toward a final length and that both the growth velocity and final depth of the invasion structures increase with increasing injection pressure. The initial constant growth rate seems to scale with the injection pressure as $P_{\mathrm{in}}^{\frac{3}{2}}$. In Fig. 12(b), the finger tip positions per category is divided by $P_{\text {in }}^{\frac{3}{2}}$ to show that they fall along the same line initially. In the simulations done by 

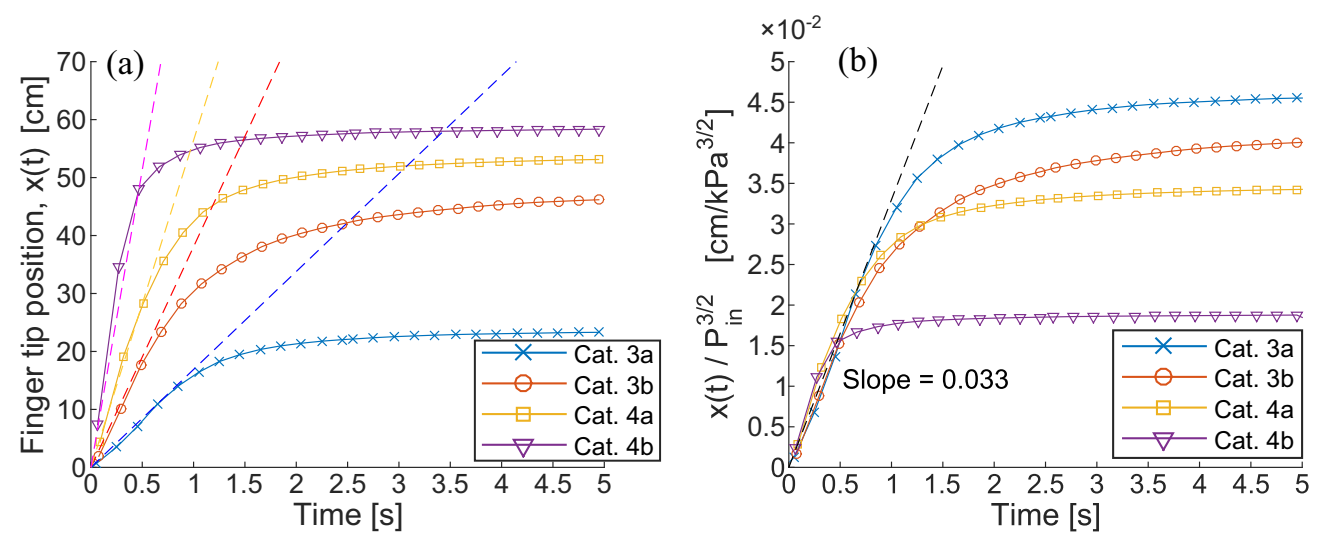

FIG. 12. (a) The finger tip position $x(t)$ as function of time, averaged for all experiments per category. The curves typically have an initial linear growth with time which crosses over to a decay with time. The patterns grow faster and longer for increasing injection pressure, and the initial linear growth is found to scale proportionally with $P_{\text {in }}^{3 / 2}$ as indicated by the dashed lines. (b) The finger tip positions $x(t)$ divided by $P_{\text {in }}^{\frac{3}{2}}$, showing that the initial growth fall along the same line. The injection pressures are $P_{\text {in }}=64,110,134$, and $213 \mathrm{kPa}$, for categories $3 \mathrm{a}$, $3 \mathrm{~b}$ and $4 \mathrm{a}, 4 \mathrm{~b}$, respectively.

Niebling et al. $[47,55]$ of a similar but smaller system, they too observe an initial linear growth velocity crossing over to decay over time; however, we here observe the initial growth velocity to scale with $P_{\text {in }}$ as $v \propto P_{\text {in }}^{3 / 2}$ instead of $v \propto P_{\text {in }}^{1 / 2}$ as found in these simulations. In Fig. 13(a), the log-log plot of the finger tip velocity $v(t) /\left(P_{\mathrm{in}}^{3 / 2}\right)$ as function of time indicates that when the finger tip velocity begins to decrease, it follows a power-law equation with time, $v(t) \propto t^{-\alpha}$, where $\alpha=2.5 \pm 0.2$.

\section{E. Scaled growth model}

As we have shown in Figs. 12 and 13, the growth of the channel length is linear with time initially until it crosses over to decay toward a final channel length. More specifically, the growth velocity is constant initially, before it crosses over to a power-law decay with time. The constant initial velocity $v_{0}$ is found to scale with injection pressure $P_{\text {in }}$ as

$$
v_{0}=C P_{\mathrm{in}}^{\frac{3}{2}}
$$

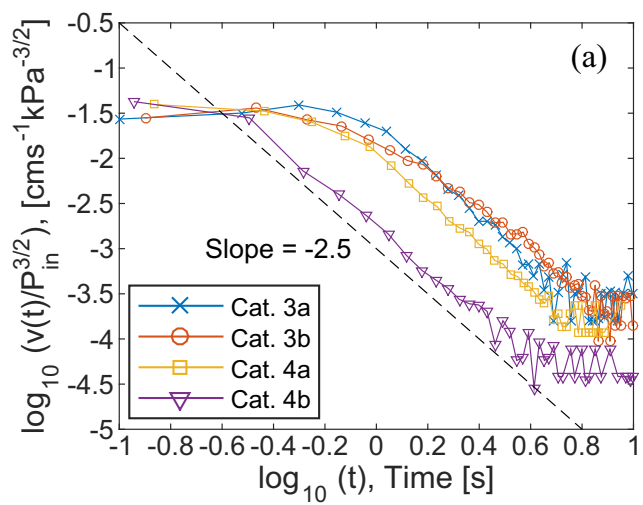

where $C \approx 10^{-\frac{3}{2}} \mathrm{~cm} /\left(\mathrm{s} \mathrm{kPa}^{\frac{3}{2}}\right)$ is found from the initial part of the collapsed curves $v(t) / P_{\text {in }}^{\frac{3}{2}}$ along the ordinate axis in Fig. 13(a). By inserting the value for $C$ into Eq. (7), we can instead collapse the curves along the ordinate axis with $v(t) / v_{0}$, resulting in dimensionless and normalized units for the velocity. In order to collapse the curves along the time axis, we define a critical time $t=t_{c}$ as the time when the growth velocity crosses over from being constant to follow a power law decay with time. At the critical time, the finger tip position can be described mathematically as

$$
x_{c}=x\left(t_{c}\right)=v_{0} t_{c},
$$

which gives the relation

$$
t_{c}=\frac{x_{c}}{v_{0}}=\frac{x_{c}}{C P_{\mathrm{in}}^{\frac{3}{2}}}
$$

for the critical time.

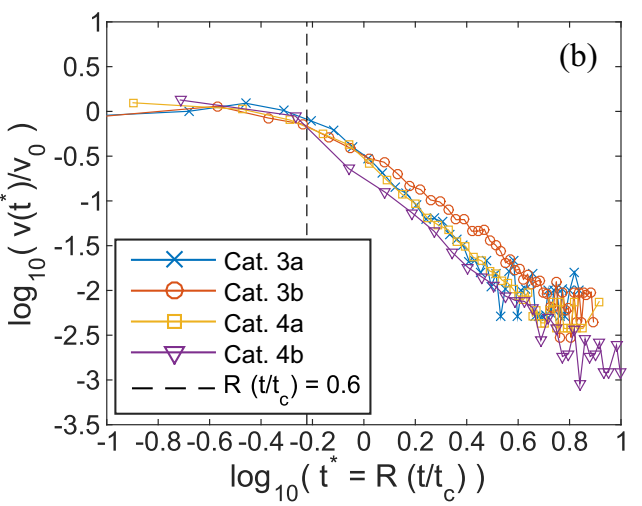

FIG. 13. (a) Log-log plot of the finger tip velocity $v(t)$ as function of time, averaged per category. The data is collapsed along the ordinate axis by dividing $v(t)$ by $P_{\mathrm{in}}^{\frac{3}{2}}$, where $P_{\text {in }}=64,110,134$, and $213 \mathrm{kPa}$, for categories $3 \mathrm{a}, 3 \mathrm{~b}$ and $4 \mathrm{a}, 4 \mathrm{~b}$, respectively. The initial constant plateau corresponds to the period with linear growth over time, which then crosses-over to a declining slope close to -2.5 (dashed line) for all the categories. The slopes show that the decay in growth velocity follows a power law equation with time. (b) The curves of normalized finger tip velocity $v\left(t^{*}\right) / v_{0}$ are collapsed along the time axis by scaling the time as $t^{*}=t\left(v_{0} / x_{f}\right)=R\left(t / t_{c}\right)$, where $x_{f}$ is the final channel length. The knee point of the curves, $R \approx 0.6$, is marked by the dashed vertical line. 



FIG. 14. Left: The collapsed finger tip positions $x^{\prime}=x\left(t^{\prime}\right) / v_{0} t_{c}$ as function of $t^{\prime}=t / t_{c}$, for individual experiments with various injection pressure. The dotted vertical line indicates the cross-over at $t^{\prime}=1$, and the dashed curve shows the proposed function $f_{x}\left(t^{\prime}\right)$. Right: Log-log plot of the collapsed finger tip velocities $v^{\prime}=v\left(t^{\prime}\right) / v_{0}$ as function of $t^{\prime}=t / t_{c}$ for the same individual experiments. The dotted vertical line indicates the cross-over at $t^{\prime}=1$, and the dashed curve shows the proposed function $f_{v}\left(t^{\prime}\right)$. Recall that the parameters used for the collapse are given by the injection pressure, as $v_{0}=C P_{\text {in }}^{\frac{3}{2}}$ and $t_{c}=[(\alpha-1) / \alpha)\left(x_{f} / v_{0}\left(P_{\text {in }}\right)\right]$, where $C=10^{-\frac{3}{2}} \mathrm{~cm} /\left(\mathrm{s} \mathrm{kPa}^{\frac{3}{2}}\right)$ and $\alpha=-2.5$.

Since we can easily measure $x_{f}$, the final length of the channel, we check if $x_{c}$ can be described as a typical fraction $R$ of $x_{f}$, such that $x_{c}=R x_{f}$, which gives

$$
t_{c}=\frac{R x_{f}}{C P_{\mathrm{in}}^{\frac{3}{2}}}=R \frac{x_{f}}{v_{0}} .
$$

By plotting $v\left(t^{*}\right) / v_{0}$ as function of $t^{*}=t\left(v_{0} / x_{f}\right)=R\left(t / t_{c}\right)$ in a log-log plot, we do indeed find that the curves collapse along the time axis with a crossover point corresponding to $R \approx 0.6$ [read from the knee point in Fig. 13(b)]. Since we now have the constants $C$ and $R$, we can calculate $v_{0}$ and $t_{c}$ for individual experiments by inserting the respective $P_{\text {in }}$ and $x_{f}$ into Eqs. (7) and (10). Figure 14 shows $\log -\log$ plots of $v^{\prime}=$ $v\left(t^{\prime}\right) / v_{0}$ as function of $t^{\prime}=t / t_{c}$ for individual experiments with injection pressures in the range of 50-250 $\mathrm{kPa}$, which follow the same dimensionless curve given by the function

$$
f_{v}\left(t^{\prime}\right)= \begin{cases}1, & \text { if } t^{\prime} \leqslant 1 \\ t^{\prime-\alpha}, & \text { if } t^{\prime}>1, \text { where } \alpha=2.5 .\end{cases}
$$

Similarly, we collapse the fingertip position $x^{\prime}=x\left(t^{\prime}\right) / x_{c}$ and plot it as function of $t^{\prime}=t / t_{c}$ for the same individual experiments, also shown in Fig. 14 (Note that the data is from single experiments, i.e., not the average values as in Fig. 13, explaining the increased amount of noise). Here, the collapsed data follow the curve given by the function

$$
f_{x}\left(t^{\prime}\right)= \begin{cases}t^{\prime}, & \text { if } t^{\prime} \leqslant 1, \\ \frac{1}{1-\alpha} t^{\prime 1-\alpha}+\frac{\alpha}{\alpha-1}, & \text { if } t^{\prime}>1,\end{cases}
$$

which is obtained by integrating each part of Eq. (11), requiring that $f_{x}\left(t^{\prime}>1\right) \rightarrow 1$ when $t^{\prime} \rightarrow 1$. Further, $f_{x}(\infty)=\alpha /(\alpha-$ $1)=x_{f} / x_{c}=1 / R$, giving $R=(\alpha-1) / \alpha=0.6$, which fits very well with our observation in Fig. 13(b). By substituting $\alpha$ into Eq. (10), we get

$$
t_{c}=\frac{\alpha-1}{\alpha} \frac{x_{f}}{C P_{\mathrm{in}}^{\frac{3}{2}}} .
$$

With Eqs. (7)-(13), we propose a description of the fundamental dynamics of the system. Since we have experimentally estimated $\alpha$ and Eq. (7) relating the initial growth velocity to injection pressure, we can estimate the evolution of the channel length during the invasion for a given injection pressure and final channel length.

\section{DISCUSSION AND CONCLUSION}

The channel formations seen in our experiments result in patterns very similar to other natural patterns arising from Laplacian growth, e.g., DLA clusters, viscous fingers in empty Hele-Shaw cells, manganese dendrites, or lightning bolts. In such systems, ramified structures expand at a rate proportional to the gradient of a Laplacian $\left(\nabla^{2} \phi=0\right)$ potential field. This type of pattern is in the DLA universality class, where a fractal dimension of $D=1.71$ is expected $[42,46]$. However, the fractal dimensions found for our patterns mainly take values of $D \in$ [1.53-1.60], which is more similar to viscous fingers in porous Hele-Shaw cells $[42,46]$. It has been established that flow in porous media is better described by another Laplacian model, i.e., the dielectric breakdown model (DBM), where the interfacial growth rate is proportional to the pressure gradient of a power $\eta$ higher than 1 , i.e., $v \propto(\nabla P)^{\eta}$, where $\eta=2$ for viscous fingers in porous media $[42,46]$. In our system, the potential field is the overpressure in the medium, which diffuses into the medium initially (not Laplacian). However, numerical simulations show that the diffusing pressure field quickly approaches the Laplace solution outside finger tips (after 0.4-0.5 s), and in the entire cell on the order of a second [28]. In addition, we observe a feature of the channel growth which is typical for Laplacian growth systems, i.e., there is an active growth zone outside a frozen structure, due to screening of the potential gradient by the most advanced parts of the structure.

The resulting channels in our experiments are different than channels formed in similar systems having open outer boundaries, such as in Refs. [24-26]. In the open systems, the channels are generally much smoother and does not have more than one or two branches, while in the closed system we see ramified structures. This is thought to be a consequence of 
the outer boundary conditions, which directly influence how the medium can be deformed. In both systems, the beads are compacted and pushed in front of the growing channel, but in the open system there is also a decompaction front moving inward from the open outlet, easing the further displacement of beads. On the other hand, in the closed system, after the compaction front hits the outer boundary (which happens between $t=150-300 \mathrm{~ms}$ [28]) further deformation is more difficult due to compaction. Thus, the local configuration of the pressure field on the tips could play a larger role on where the channels grow in this case. In addition, the overpressures imposed for channeling in our experiments ( $P_{\text {in }}=40-250 \mathrm{kPa}$ ) are one order of magnitude larger than the ones for these open systems $\left(P_{\text {in }}=2-20 \mathrm{kPa}\right)$. Although the cells in Refs. [24,26] are smaller than ours, the one in Ref. [25] is of comparable dimensions.

The average fluid drag per unit area on the granular medium of height $h$ is given by $\nabla P h$, and the average threshold for bead displacement is given by [25]

$$
\sigma_{\text {yield }}=\mu(1-\phi) \rho g h=\mu \rho_{s} \rho g h,
$$

where $\mu$ is the Coulomb friction coefficient, $\rho_{s}$ is the solid fraction of the granular medium, $\rho$ is the density of the bead material, and $g$ is the acceleration of gravity. Equation (14) is found by considering the weight and friction of the granular medium on the bottom plate while neglecting forces transmitted through solid contacts, i.e., in-plane stress and friction at the top plate, which is reasonable for the initial medium. Then, we have the requirement for bead displacement as

$$
\nabla P h \geqslant \sigma_{\text {yield }} \Rightarrow \nabla P \geqslant \mu \rho_{s} \rho g,
$$

which indicates that the pressure gradient necessary to deform the initial granular medium increases with the friction coefficient, solid fraction, and the bulk density of beads. In a system where the injected fluid can be considered compressible (like air in our experiment), the overpressure diffuses into the medium with a diffusion constant $[28,47]$

$$
D=\frac{\kappa}{\left(1-\rho_{s}\right) c \mu_{f}}=\frac{d^{2}\left(1-\rho_{s}\right)^{2}}{180 \rho_{s} c \mu_{f}},
$$

where $\kappa$ is the permeability estimated with the CarmanKozeny expression, $d$ is the bead diameter, $c$ is the fluid compressibility, and $\mu_{f}$ is the fluid viscosity. By assuming a solid fraction close to 0.5 for the initial medium, we see that the diffusivity of the overpressure is related to the bead size as

$$
D \sim \frac{d^{2}}{c \mu_{f}} .
$$

Thus, the bead size and cell length influence whether the fluid injection acts as a body force on the medium or more like a surface force on the air-solid interface; i.e., channels do not form with large enough beads or short enough cell because the effective range of the pressure gradient (skin depth) exceeds the system length, such that beads are primarily displaced by the fluid [47]. Smaller beads or longer cells lead to a more localized skin depth such that beads are primarily displaced through solid contacts (the zone of mobilized beads eventually exceeds the skin depth) and channels form.
Further, the cell length limits the maximum length of eventual channels formed [28] (longer cells accommodate longer channels). A wider cell leads to an increase in the average number of fingers [47] but not necessarily an increase in the finger width $[25,26]$. However, the channels could become thicker with increased cell width for flow category $4 \mathrm{~b}$ since a higher number of fingers could merge together. A decrease in cell gap has a similar effect as an increase in cell width, which leads to a higher number of fingers, and an increase in the cell gap above $2 \mathrm{~mm}$ usually introduces 3D effects where the fluid tends to flow mostly in the top layer of the granular medium and to displace grains preferentially there.

For the growth dynamics, the dependency of $v_{0}$ on $P_{\text {in }}$ and the exponent $\alpha$ could be system dependent; i.e., the parameters could depend on, e.g., bead size and confinement. Thus, it is of interest to investigate further how the dynamics are changing in various confined granular media, and see how the Eqs. (7)-(13) apply in those cases. The dependency of $x_{f}$ on $P_{\text {in }}$ is also of interest to investigate further, such that if system-dependent parameters are known, the expected result of an air injection could be estimated by the injection pressure alone. A brief discussion of what we could find from our experiments is presented in Fig. 15. Another interesting feature found to be common for all experiments in the system is the typical profile of the finger tips, corresponding to a growth in finger thickness $w$ as function of distance $d$ behind the tip as $w \propto d^{\beta}$, where $\beta=0.68$ on average.

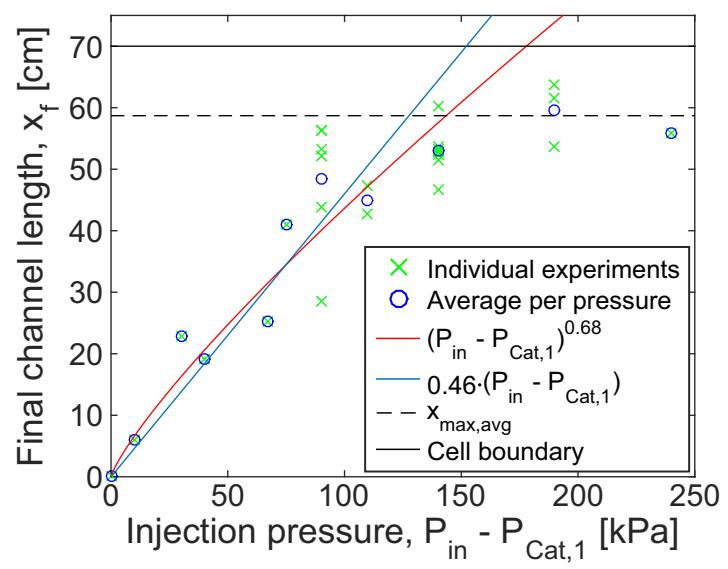

FIG. 15. The final channel length $x_{f}$ is plotted as function of $P_{\text {in }}-P_{\text {cat }, 1}$, where $P_{\text {cat }, 1}=9 \mathrm{kPa}$ is the average injection pressure for category 1 , where $x_{f}=0$. We see that there is a rather scattered (due to randomness in the initial bead configuration from experiment to experiment), but increasing trend in $x_{f}$ for increasing $P_{\text {in }}$, but also that the final channel length for the highest injection pressures is limited by the closed outer boundary. The fit $\left(P_{\text {in }}-P_{\text {cat }, 1}\right)^{0.68}$ was found from the averaged $x_{f}$ per pressure for $P_{\text {in }} \in[20,150] \mathrm{kPa}$ and suggest a powerlaw relationship. Alternatively, the linear fit $0.46 \cdot\left(P_{\text {in }}-P_{\text {cat }, 1}\right)$ fits equally well. The average limiting line $x_{\max , a v g}$ was found by averaging $x_{f}$ over the experiments with $P_{\text {in }} \geqslant 200 \mathrm{kPa}$, to indicate the influence of the cell boundary. Thus, the results from our experiments indicate that $x_{f}$ increase more or less proportionally with $P_{\text {in }}$ until a limiting length $x_{\text {max,avg }} \approx 59 \mathrm{~cm}$ is reached due to the presence of the outer boundary. 
As shown in Fig. 11, the fractal dimension of the channels is found to be fairly stable along the main part of the structures, even for the thick fingers in category $4 \mathrm{~b}$. The local box-counting method indicates that when there is little or no erosion, as is the case for categories $3 \mathrm{a}, 3 \mathrm{~b}$, and $4 \mathrm{a}$, the final structures end up with typical fractal dimensions between $D=1.53$ and 1.60. This range is similar to the one observed for viscous fingers in saturated porous media, i.e., $D=1.53-1.62$, suggesting that the channels formed in our experiments are in the DBM universality class of patterns where the interface expands at a rate proportional to $(\nabla P)^{2}$. The thicker category $4 \mathrm{~b}$ structures have a fractal dimension of $D=1.76$, which is closer to DLA-patterns and viscous fingering in empty saturated Hele-Shaw cells $(D=1.71)$. However, the higher fractal dimension is probably also an effect of box-counting inside the main channel, which is rather space filling compared to the system size, and this makes the box-counting slopes for category $4 \mathrm{~b}$ more uncertain than for thinner fingers. Furthermore, the global box-counting dimensions $D_{B}$ are slightly lower than the locally estimated fractal dimensions, which could be a finite-size effect since less developed outer parts are also taken into account. However, for the most developed and best preserved channels (categories $3 \mathrm{~b}$ and $4 \mathrm{a}$ ), we find that $D_{B}=1.53$ and 1.54 , which is still in good agreement with the fractal dimensions for viscous fingers in porous media. Finally, the fractal dimensions of the front is found to lie between $D_{F}=1.41$ and 1.52 for all categories. This indicates that even if the structures themselves appear to change with increasing injection pressure, the roughness of the air-solid interface seems to be more or less the same.

\section{ACKNOWLEDGMENTS}

This project has received funding from the European Union's Seventh Framework Programme for research, technological development, and demonstration under Grant Agreement No. 316889, ITN FlowTrans, from the University of Strasbourg through an "Espoirs" grant, from the CNRS-INSU through an "ALEAS" grant, and from the LIA France Norway D-FRACT. This work was partly supported by the Research Council of Norway through its Centres of Excellence funding scheme, Project No. 262644. We thank Alain Steyer and Miloud Talib for their technical support with experimental equipment. We also thank Emily Brodsky, Daniel Koehn, Piotr Szymczak, Christine Putnis, Ulrich Kelka, Stephen Centrella, Amir Sagy, Monem Ayaz, Anke Lindner, Harold Auradou, Bjørnar Sandnes, and Øistein Johnsen for fruitful discussions.
[1] S. S. Suthersan, Remediation Engineering: Design Concepts (CRC Press LLC, Boca raton, FL, 1999), pp. 237-54.

[2] L. L. Goodroad and J. R. Schuring, Potential of pneumatic fracturing to enhance in situ remediation technologies, in Proceedings of the Waste Management 1994 Symposium (Tucson, AZ, 1994).

[3] S. Kazemian, B. B. K. Huat, A. Prasad, and M. Barghchi, A review of stabilization of soft soils by injection of chemical grouting, Aust. J. Basic Appl. Sci. 4, 5862 (2010).

[4] R. Z. Moayed, E. Izadi, and M. Fazlavi, In situ stress measurements by hydraulic fracturing method at Gotvand dam site, Iran, Turkish J. Eng. Env. Sci. 36, 179 (2012).

[5] C. T. Montgomery and M. B. Smith, Hydraulic fracturing: history of an enduring technology, J. Pet. Technol. 62, 26 (2010).

[6] Department of Energy, Hydraulic fracturing whitepaper, EPA 816-R-04-003, Appendix A (2004).

[7] G. C. Naik, Tight gas reservoirs-An unconventional natural energy source for the future, retrieved from www.pinedaleonline. com/socioeconomic/pdfs/tight_gas.pdf (2003).

[8] S. M. Johnson and J. P. Morris, Hydraulic fracturing mechanisms in carbon sequestration applications, in 43rd U.S. Rock Mechanics Symposium and 4th U. S.-Canada Rock Mechanics Symposium, (American Rock Mechanics Association, Asheville, NC, 2009).

[9] W. H. Williamson and D. R. Wooley, Hydraulic Fracturing to Improve the Yield of Bores in Fractured Rock (Australian Government Publishing Service, Canberra, ACT, 1980).

[10] F. Rummel and O. Kappelmayer, The Falkenberg geothermal frac-project: Concepts and experimental results, Hydraul. Fract. Geotherm. Energy 5, 59 (1983).

[11] L. Clark, Energy company plans to frack volcano, in Wired Science, retrieved from www.wired.com/wiredscience/2012/10/ newberry-volcano-fracking (2012).
[12] A. Hurst, J. Cartwright, and D. Duranti, in Fluidization Structures Produced by Upward Injection of Sand Through a Sealing Lithology, edited by P. Van Rensbergen, R. R. Hillis, A. J. Maltman, and C. K. Morley, Subsurface Sediment Mobilization, Special Publications 216 (Geological Society, London, 2003), pp. 123-138.

[13] H. Løseth, L. Wensaas, B. Arntsen, and M. Hovland, in Gas and Fluid Injection Triggering Shallow Mud Mobilization in the Hordaland Group, North Sea, edited by P. Van Rensbergen, R. R. Hillis, A. J. Maltman, and C. K. Morley, Subsurface Sediment Mobilization, Special Publications 216 (Geological Society, London, 2003), pp. 139-157.

[14] P. Van Rensbergen, J. Poort, R. Kipfer, M. De Batist, M. Vanneste, J. Klerkx et al., Near-surface Sediment Mobilization and Methane Venting in Relation to Hydrate Destabilization in Southern Lake Baikal, Siberia, edited by P. Van Rensbergen, R. R. Hillis, A. J. Maltman, and C. K. Morley, Subsurface Sediment Mobilization, Special Publications 216 (Geological Society, London, 2003), pp. 207-221.

[15] A. R. Talukder, M. C. Comas, and J. I. Soto, Pliocene to Recent Mud Diapirism and Related Mudvolcanoes in the Alboran Sea (Western Mediterrenean), edited by P. Van Rensbergen, R. R. Hillis, A. J. Maltman, and C. K. Morley, Subsurface Sediment Mobilization, Special Publications 216 (Geological Society, London, 2003), pp. 443-459.

[16] N. Pralle, M. Külzer, and G. Gudehus, Experimental Evidence on the Role of Gas in Sediment Liquefaction and Mudvolcanism, edited by P. Van Rensbergen, R. R. Hillis, A. J. Maltman, and C. K. Morley, Subsurface Sediment Mobilization, Special Publications 216 (Geological Society, London, 2003), pp. 159-171.

[17] E. Deville, A. Battani, R. Griboulard, S. Guerlais, J. P. Herbin, J. P. Houzay et al., The Origin and Processes of Mudvolcanism: 
New Insights from Trinidad, edited by P. Van Rensbergen, R. R. Hillis, A. J. Maltman, and C. K. Morley, Subsurface Sediment Mobilization, Special Publications 216 (Geological Society, London, 2003), pp. 475-490.

[18] R. Nuwer, Indonesia's "mud volcano" and nine years of debate about its muck, retrieved from www.nytimes.com/2015/09/22/ science/9-years-of-muck-mud-and-debate-in-java.html (2015).

[19] A. Mazzini, H. Svensen, G. G. Akhmanov, G. Aloisi, S. Planke, A. Malthe-Sørenssen, and B. Istadi, Triggering and dynamic evolution of the Lusi mud volcano, Indonesia, Earth Planet. Sci. Lett. 261, 375 (2007).

[20] A. Mazzini, A. Nermoen, M. Krotkiewski, Y. Podladchikov, S. Planke, and H. Svensen, Strike-slip faulting as a trigger mechanism for overpressure release by piercement structures. implications for the Lusi mud volcano, Indonesia, Marine Petrol. Geol. 26, 1751 (2009).

[21] A. Mazzini, G. Etiope, and H. Svensen, A new hydrothermal scenario for the 2006 Lusi eruption, indonesia. insights from gas geochemistry, Earth Planet. Sci. Lett. 317/318, 305 (2012).

[22] M. Lupi, E. H. Saenger, F. Fuchs, and S. A. Miller, Lusi mud eruption triggered by geometric focusing of seismic waves, Nature Geosci. 6, 642 (2013).

[23] M. R. P. Tingay, M. L. Rudolph, M. Manga, R. J. Davies, and C. Y. Wang, Initiation of the Lusi mudflow disaster, Nature Geosci. 8, 493 (2015).

[24] Ø. Johnsen, R. Toussaint, K. J. Måløy, and E. G. Flekkøy, Pattern formation during air injection into granular materials confined in a circular hele-shaw cell, Phys. Rev. E 74, 011301 (2006).

[25] Ø. Johnsen, R. Toussaint, K. J. Måløy, E. G. Flekkøy, and J. Schmittbuhl, Coupled air/granular flow in a linear hele-shaw cell, Phys. Rev. E 77, 011301 (2008).

[26] Ø. Johnsen, C. Chevalier, A. Lindner, R. Toussaint, E. Clément, K. J. Måløy, E. G. Flekkøy, and J. Schmittbuhl, Decompaction and fluidization of a saturated and confined granular medium by injection of a viscous liquid or gas, Phys. Rev. E 78, 051302 (2008).

[27] M. J. Niebling, E. G. Flekkøy, K. J. Måløy, and R. Toussaint, Sedimentation instabilities: Impact of the fluid compressibility and viscosity, Phys. Rev. E 82, 051302 (2010).

[28] F. K. Eriksen, R. Toussaint, A. L. Turquet, K. J. Måløy, and E. G. Flekkøy, Pressure evolution and deformation of confined granular media during pneumatic fracturing (unpublished).

[29] X. Cheng, L. Xu, A. Patterson, H. M. Jaeger, and S. R. Nagel, Toward the zero-surface-tension limit in granular fingering instability, Nat. Phys. 4, 234 (2008).

[30] P. G. Saffman and G. Taylor, The penetration of a fluid into a porous medium or hele-shaw cell containing a more viscous liquid, Proc. R. Soc. Lond. A. Math. Phys. Sci. 245, 312 (1958).

[31] F. K. Eriksen, R. Toussaint, K. J. Måløy, and E. G. Flekkøy, Invasion patterns during two-phase flow in deformable porous media, Front. Phys. 3, 81 (2015).

[32] B. Sandnes, E. G. Flekkøy, H. A. Knudsen, and K. J. Måløy, Patterns and flow in frictional fluid dynamics, Nat. Commun. 2, 288 (2011).

[33] R. Holtzman, M. L. Szulczewski, and R. Huanes, Capillary Fracturing in Granular Media, Phys. Rev. Lett. 108, 264504 (2012).
[34] X. Z. Kong, W. Kinzelbach, and F. Stauffer, Morphodynamics during air injection into water-saturated movable spherical granulates, Chem. Eng. Sci. 65, 4652 (2010).

[35] C. Chevalier, A. Lindner, M. Leroux, and E. Clément, Morphodynamics during air injection into a confined granular suspension, J. Non-Newton Fluid Mech. 158, 63 (2008).

[36] J. A. Eriksen, B. Marks, B. Sandnes, and R. Toussaint, Bubbles breaking the wall: Two-dimensional stress and stability analysis, Phys. Rev. E 91, 052204 (2015).

[37] J. A. Eriksen, R. Toussaint, K. J. Måløy, E. G. Flekkøy, and B. Sandnes, Numerical approach to frictional fingers, Phys. Rev. E 92, 032203 (2015).

[38] B. Marks, B. Sandnes, G. Dumazer, J. A. Eriksen, and K. J. Måløy, Compaction of granular material inside confined geometries, Front. Phys. 3, 41 (2015).

[39] J. A. Eriksen, R. Toussaint, K. J. Måløy, E. G. Flekkøy, and B. Sandnes, Pattern formation of frictional fingers in a gravitational potential, arXiv:1605.07436v1 (2016).

[40] M. Moura, E. A. Fiorentino, K. J. Måløy, G. Schäfer, and R. Toussaint, Impact of sample geometry on the measurement of pressure-saturation curves: Experiments and simulations, Water Resour. Res. 51, 8900 (2015).

[41] D. Wilkinson and J. F. Willemsen, Invasion percolation: A new form of percolation theory, J. Phys. A Math. Gen. 16, 3365 (1983).

[42] G. Løvoll, Y. Méheust, R. Toussaint, J. Schmittbuhl, and K. J. Måløy, Growth activity during fingering in a porous hele-shaw cell, Phys. Rev. E 70, 026301 (2004).

[43] G. Løvoll, M. Jankov, K. J. Måløy, R. Toussaint, J. Schmittbuhl, G. Schäfer, and Y. Méheust, Influence of viscous fingering on dynamic saturation-pressure curves in porous media, Transp. Porous Med. 86, 305 (2011).

[44] K. T. Tallakstad, H. A. Knudsen, T. Ramstad, G. Løvoll, K. J. Måløy, R. Toussaint, and E. G. Flekkøy, Steady-State Two-Phase Flow in Porous Media: Statistics and Transport Properties, Phys. Rev. Lett. 102, 074502 (2009).

[45] H. Huang, F. Zhang, P. Callahan, and J. Ayoub, Granular Fingering in Fluid Injection into Dense Granular Media in a Hele-Shaw Cell, Phys. Rev. Lett. 108, 258001 (2012).

[46] R. Toussaint, G. Løvoll, Y. Méheust, K. J. Måløy, and J. Schmittbuhl, Influence of pore-scale disorder on viscous fingering during drainage, Europhys. Lett. 71, 583 (2005).

[47] M. J. Niebling, R. Toussaint, E. G. Flekkøy, and K. J. Måløy, Dynamic aerofracture of dense granular packings, Phys. Rev. E 86, 061315 (2012).

[48] M. J. Niebling, E. G. Flekkøy, K. J. Måløy, and R. Toussaint, Mixing of a granular layer falling through a fluid, Phys. Rev. E 82, 011301 (2010).

[49] J. L. Vinningland, Ø. Johnsen, E. G. Flekkøy, R. Toussaint, and K. J. Måløy, Granular Rayleigh-Taylor Instability: Experiments and Simulations, Phys. Rev. Lett. 99, 048001 (2007).

[50] J. L. Vinningland, Ø. Johnsen, E. G. Flekkøy, R. Toussaint, and K. J. Måløy, Experiments and simulations of a gravitational granular flow instability, Phys. Rev. E 76, 051306 (2007).

[51] J. L. Vinningland, Ø. Johnsen, E. G. Flekkøy, R. Toussaint, and K. J. Måløy, Size invariance of the granular Rayleigh-Taylor instability, Phys. Rev. E 81, 041308 (2010).

[52] J. L. Vinningland, R. Toussaint, M. J. Niebling, E. G. Flekkøy, and K. J. Måløy, Family-vicsek scaling of detachment fronts 
in granular Rayleigh-Taylor instabilities during sedimentating granular/fluid flows, Eur. Phys. J. Special Topics 204, 27 (2012).

[53] C. Völtz, W. Pesch, and I. Rehberg, Rayleigh-taylor instability in a sedimenting suspension, Phys. Rev. E 65, 011404 (2001).

[54] H. M. Jaeger, S. R. Nagel, and R. P. Behringer, Granular solids, liquids, and gases, Rev. Mod. Phys. 68, 1259 (1996).

[55] M. J. Niebling, R. Toussaint, E. G. Flekkøy, and K. J. Måløy, Numerical studies of aerofractures in porous media, Rev. Cub. Fis. 29, 1E66 (2012).

[56] S. Turkaya, R. Toussaint, F. K. Eriksen, M.Zecevic, G. Daniel, E. G. Flekkøy, and K. J. Måløy, Bridging aero-fracture evolution with the characteristics of the acoustic emissions in a porous medium, Front. Phys. 3, 70 (2015).

[57] J. Ugelstad, P. C. Mórk, K. H. Kaggerud, T. Ellingsen, and A. Berge, Swelling of oligomer-polymer particles: New methods of preparation, Adv. Colloid Interface Sci. 13, 101 (1980); Produced under the trade name Dynospheres by Dyno Particles A.S., N-2001 Lillestrom, Norway.
[58] D. R. Nelson, Order, frustration, and two-dimensional glass, in Topological Disorder in Condensed Matter, edited by F. Yonesawa and T. Ninomiya, Springer Series in Solid-State Sciences (Springer, Berlin, 1983), Vol. 46, pp. 164-180.

[59] See Supplemental Material at http://link.aps.org/supplemental/ 10.1103/PhysRevE.95.062901 for the full-size figure of final structures for each category; a video of a Cat. 3 a channel ( $P_{\text {in }}=$ $50 \mathrm{kPa}$, video is $0.25 \times$ realtime); a video of a Cat. $3 \mathrm{~b}$ channel ( $P_{\text {in }}=100 \mathrm{kPa}$, video is $0.25 \times$ realtime); a video of a Cat. $4 \mathrm{a}$ channel ( $P_{\text {in }}=150 \mathrm{kPa}$, video is $0.25 \times$ realtime); a video of a Cat. $4 \mathrm{~b}$ channel $\left(P_{\text {in }}=250 \mathrm{kPa}\right.$, video is $0.05 \times$ realtime $)$; the full-size version of figure 6 , showing erosion and fingers merging in an experiment with $P_{\text {in }}=200 \mathrm{kPa}$.

[60] A. L. Barabási and H. E. Stanley, Fractal Concepts in Surface Growth (Cambridge University Press, Cambridge, 1995).

[61] J. Feder, Fractals (Plenum Press, New York, 1988).

[62] B. B. Mandelbrot, The Fractal Geometry of Nature (W.H. Freeman and Company, New York, 1982).

[63] K. J. Måløy, J. Feder, and T. Jøssang, Viscous Fingering Fractals in Porous Media, Phys. Rev. Lett. 55, 2688 (1985). 